\title{
Improvement of Community Health Outcomes in Kitgum District: Contributions of a Maternal Newborn and Child Health Project from June 2011 to July 2016
}

\author{
Babughirana Geoffrey ${ }^{1}$, Saul Onyango ${ }^{2}$, Lorna Muhirwe Barungi ${ }^{1}$, Irene Mbugua ${ }^{1}$, \\ Benon Musasizi ${ }^{1}$, Edgar Twinomujuni Rukambura ${ }^{1}$ \\ ${ }^{1}$ World Vision, Kampala, Uganda \\ ${ }^{2}$ Independent Scholar, Kampala, Uganda
}

Email address:

gbabughirana@gmail.com (B. Geoffrey), sonyango@gmail.com (S. Onyango), lornamuhirwe@gmail.com (L. M. Barungi), irenembugua2@gmail.com (I. Mbugua), musasizi.benon1@gmail.com (B. Musasizi),twinruk@gmail.com (E. T. Rukambura)

\section{To cite this article:}

Babughirana Geoffrey, Saul Onyango, Lorna Muhirwe Barungi, Irene Mbugua, Benon Musasizi, Edgar Twinomujuni Rukambura. Improvement of Community Health Outcomes in Kitgum District: Contributions of a Maternal Newborn and Child Health Project from June 2011 to July 2016. Science Journal of Public Health. Vol. 5, No. 1, 2017, pp. 10-19. doi: 10.11648/j.sjph.20170501.12

Received: November 17, 2016; Accepted: December 1, 2016; Published: December 26, 2016

\begin{abstract}
The East African Maternal Newborn and Child Health (EAMNCH) project implemented in Kitgum District of Uganda with a goal to improve $\mathrm{MNCH}$. The evaluation was conducted as a cross-sectional assessment that utilised both quantitative and qualitative data collection methods. The projects contribution to the goal included a $4 \%$ reduction in stunting levels, $5.9 \%$ increase in the number of women delivered by a skilled Birth attendant, $12.6 \%$ increment in pregnant women accessing Iron folic supplements and a 7\% increment in utilization of ITN during pregnancy. Evidence from the quantitative household survey, key informant interviews and focus group discussions supported the contribution of project interventions to the reduction in infant and under-five child morbidity, reduction in maternal morbidity as well as mortality within the project areas.
\end{abstract}

Keywords: Community Health Outcomes, Maternal Newborn, Child Health, Health Systems Strengthening

\section{Introduction}

\subsection{Project Area and Location}

The EAMNCH project was implemented in two sub counties of Mucwini and Lagoro (project area) found in Kitgum District in the Northern region of Uganda. The District is bordered by South Sudan to the north, Kaabong District to the east, Kotido District to the southeast, Agago District to the south, Pader District to the southwest and Lamwo District to the northwest. The district is composed of one county: Chua County and therefore one health sub district of Chua.

According to the National population and housing census 2014 report [1] the project area has a total population of 36,824 (48.4\% male and $51.2 \%$ female) with an average Annual Growth Rate of 3\%. The Project area has got an average household size of 5.3 and a total number of 7,082 households. It's located in the Post-conflict Northern
Uganda which was facing the challenges of return and resettlement of close to 1.8 million people. The two target sub counties in Kitgum district were among the worst hit areas in Northern Uganda and therefore best suited for a $\mathrm{MNCH}$ recovery project. This is where the Lord's Resistance Army (LRA) started its operations and where it committed some of the worst atrocities of the 20 year war. Hundreds of thousands of people in Kitgum were displaced and lived in IDP camps for decades. Former infrastructures were abandoned, destroyed or dilapidated, and the land was overgrown with bushes.

\subsection{The MNCH Situation in Kitgum District at the Onset of the Project}

Because of the 20 year displacement of communities, 
formerly existing community structures were eroded. Another result of the war was the high prevalence of HIV/AIDS in Kitgum, which led to many Orphans and Vulnerable Children (OVC) and child headed households. Without any parents to protect them, these children were vulnerable to all kinds of mistreatment and abuse. The teenage girls among them ran the risk of becoming pregnant, either due to rape or early marriages. Some of these OVCs were HIV positive themselves; they experience significant discrimination and face additional barriers to good $\mathrm{MNCH}$.

This was illustrated by statistical $\mathrm{MNCH}$ data in the Kitgum District Development Plan (DDP) [2]. According to the plan at that time, only $44.2 \%$ of the approved health facility posts were filled by trained staff, common causes for morbidity for children under 5: malaria 30\%, ARI $12.3 \%$, Intestinal worms $7 \%$ and malnutrition $1.8 \%$. An indicator of this was that only $17 \%$ of the households in Kitgum had a latrine, compared to $67 \%$ nationally. Women with a disability faced even more obstacles when accessing $\mathrm{MNCH}$ services. Usually there were hardly any specific facilities for them at HCs and the staff did not have the knowledge or capacity to assist them adequately. In general, people with a disability are very vulnerable to challenges related to $\mathrm{MNCH}$, such as food security and water related diseases. Traditional and cultural beliefs most often see disability as a curse from God, and people with a disability are consequently viewed with fear and prejudice and excluded from society and community life [3]. The target communities are furthermore heavily affected by the HIV pandemic. According to the Uganda Aids Atlas the HIV prevalence in Kitgum amounts to $8.2 \%$, whereby women are hit harder than men $(9 \%$ for women as compared to $7.1 \%$ for men) [4].

\subsection{The East Africa Maternal Newborn and Child Health (EAMNCH) Project}

The EAMNCH Project was designed as a five-year venture, part of the Australia-Africa Community Engagement Scheme (AACES) [5] programme for selected communities in Kenya, Rwanda, Tanzania and Uganda. The Uganda project has been implemented in Lagoro and Mucwini subcounties, Kitgum district through a partnership led by World Vision Australia with World Vision (WV) Uganda, in close collaboration with the district local government. The goal of the EAMNCH project was to improve maternal, newborn and child health in the 2 sub by ensuring a continuum of health care between households and health facilities. This was meant to be achieved by focusing on the following intervention areas: increasing access and utilization of $\mathrm{MNCH}$ services; improving food security and maternal and child nutrition; improving access to water, hygiene and sanitation; enhancing communities' knowledge of health rights and advocacy skills, and; enabling a favorable policy environment for MNCH. To increase access to and utilization of $\mathrm{MNCH}$ services the project worked on strengthening and increasing supply of $\mathrm{MNCH}$ services, while also strengthening and increasing demand for services from the community.

The project created awareness among the community and service providers on the need to strengthen the capacity of disabled women and OVC to stand up for their rights. Furthermore, ensuring that community leaders and male family members are engaged and actively supporting this project was critical to changing existing attitudes and practice in $\mathrm{MNCH}$, hygiene and sanitation. The project was carried out in close cooperation with the Ugandan Ministry of Health $(\mathrm{MoH})$. In joint efforts, solutions were sought for gaps in the health system. In the initial years of the project, WV supported many of the services offered and facilitate health workers to carry out their work. However, as the health system's capacity was be built over the years, it was expected that in the last phase of the project the government would take over those initiatives. In order to address this challenge, the project constantly engaged the $\mathrm{MoH}$ in dialogue to ensure a strong effort in building the capacity of the government system and health workers.

\subsection{Specific Objectives of the Study}

The key specific objectives of the evaluation activity were as follows:

(1) To establish if the project achieved its intended outcomes and how it had impacted the communities in the operational areas;

(2) To collect and analyse the verifiable indicators and other relevant information particularly in relation to:

i. Current status of the MNCH, nutrition, WASH, and food security;

ii. Accessibility to information and services;

iii. Civic awareness in development and governance processes in which EAMNCH operated;

(3) To provide specific, actionable and practical recommendations focusing on key sectors for future programing.

\section{Methodology}

The evaluation was conducted as a cross-sectional assessment that utilised both quantitative and qualitative data collection methods, the methodology was aligned to the ones applied during the baseline survey and mid-term review. The household survey targeting women of child bearing age (including the women with disability) as the primary respondents, with involvement of all children below five years of age in the selected households through anthropometry. Focus group discussions (FGDs) involving the following categories: i) pregnant and lactating women; ii) persons with disability; iii) male partners/ husbands; iv) Community resource persons (VHTs/CHWs); v) Parent Support Groups (PSG)

\subsection{Data Collection}

Qualitative Data: Preparation of semi-structured, openended questions for the Key Informant Interviews (KII) and 
FGD guides was done. The qualitative data collection tools were administered by Enumerators, selected on basis of having previous experience in conducting FGDs and KIIs as well as being fluent in the common local language (Acholi).

Quantitative Data: The study design comprised of the two stage cross-sectional cluster sampling methodology, with the village as the geographical unit. A structured tool was adopted from the mid-term review tool to collect quantitative data. The sample size calculations made ensured that key indicators were statistically representative of the project population and consistent with the level set during the midterm review, using the simplified formula provided by Yamane (1967:886). The confidence level was set at 95\%; the variability was set at the maximum level of 0.5 ; and the level of precision (e) was set at $\pm 4 \%$. A data screen was developed using the Open Data Kit (ODK) software for mobile platforms, which enabled data to be entered directly using a mobile phone.

\subsection{Training of Data Collection Team}

The focus on attainment of high quality findings justified the appropriate training conducted for the data collection teams, which covered technical information on $\mathrm{MNCH}$, use of the mobile platform for data collection and pilot-testing of the questionnaire. It included sessions on making correct anthropometric measurements, in line with guidelines from the ENA for SMART methodology To ensure data quality, competency exams were given to research assistants at the end of the training session and those who did not meet the set standards were excluded from the field activities.

\subsection{Data Management}

In general, analysis of the quantitative data involved summarising and preparation of frequencies and proportions as well as trends in comparision from the baseline, Midterm review (MTR) and the end line evaluation. This provided the basis for determination of the contribution of the project at outcome and output levels basing on the indicators that were agreed upon at the onset of the project as well as the national targets set out within the Health Strategic and Investment Plan (HSSIP) [6]. The anthropometric data was exported into ENA for SMART for generation of $z$-scores used to determine nutritional indicators of Weight for Height (WHZ), Weight for Age (WAZ) and Height for Age (HAZ) z-scores, using the WHO 2006 Standards. The data from household survey was analysed using IBM Statistics (SPSS) 22.

The qualitative data from FGDs was transcribed, translated in English, processed and analysed using the master sheet approach. This involved summarising and deriving the emerging themes, patterns and issues. Adequate steps were taken to ensure that quality of data was not compromised during the translation and back translation process. A consultative meeting was convened for key stakeholders to discuss the provisional findings and validate the observations.

\subsection{Ethical Consideration}

The study sought and obtained ethical approval from Makerere University School of Public Health Higher Degrees, Research and Ethics committee before it was conducted. In addition, Uganda national council for science and technology (UNCST) also approved the study under number SS 5029. Informed consent was sought from the population interviewed and other participants by signing on the administered consent form.

\subsection{End Term Evaluation (ETR) Limitations}

(1) The tool had inherent weaknesses in the type of question used to collect some data, particularly on exclusive breastfeeding and complementary feeding. Interpretation of the findings related to the above indicators should be done with caution

(2) The data collection exercise was conducted during the period just preceding national elections, with some mothers being actively involved in the political rallies and not available at home at the time the teams visited their homes.

(3) Some of the Key informants were on the electoral trail and were not readily available for the interviews.

(4) The selected villages were sparsely populated, with houses far apart and at times it was not possible to meet the targeted number of households for the day.

\section{Implementation Results}

The information presented in this chapter was based on the data collected from multiple but complementary sources as outlined in the previous chapter on methodology. The results are in comparision of the baseline to the MTR and the ETR, with explanations basing on the inputs and the World Vision 7"11 strategy, the MNCH models of choice but also on the District MNCH system's strengthening efforts as contributed by the EAMNCH project. The results are presented in an ascending order from the indicators at the goal level to those at outcome level.

\subsection{Progress Towards the EAMNCH Goal Indicators}

The overall goal of the project was to improve $\mathrm{MNCH}$ through increasing access and utilization of $\mathrm{MNCH}$ services; improving food security and maternal nutrition; improving access to WASH; improving the knowledge of communities on health rights and advocacy skills, and; enabling a favourable policy environment for $\mathrm{MNCH}$ with a Special focus on improved $\mathrm{MNCH}$ in Lagoro and Mucwini sub-counties, Kitgum District, by June 2016 as shown below by the progress towards the agreed upon Goal indicators. 


\subsubsection{Percentage Reduction in Children Aged 6-59 Months Who Are Stunted}

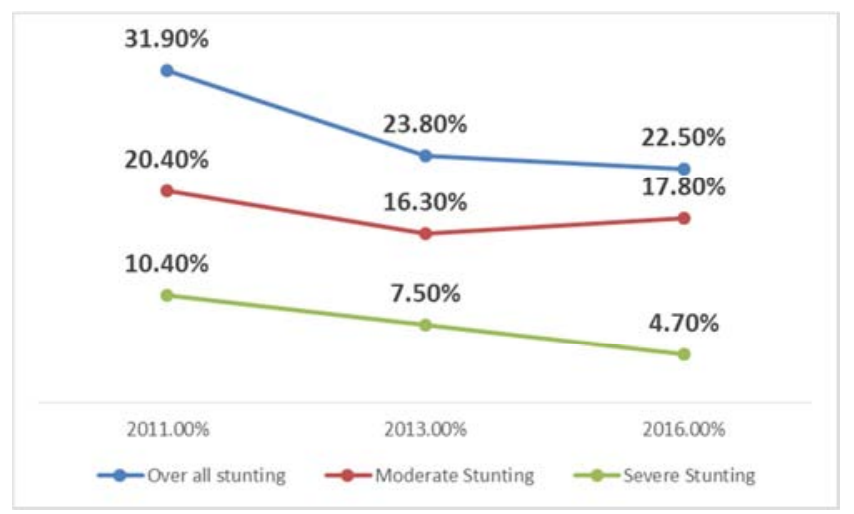

Figure 1. Trends in Prevalence of stunting in the project area.

The prevalence of stunting based on height-for-age $\mathrm{z}$ scores, among children age 6-59 months has been presented in figure 1. It revealed the overall prevalence of stunting was at $31.9 \%$ during the baseline but declined to $23.8 \%$ at MTR and further to $22.5 \%$ at ETR. This therefore presents a reduction of $9.4 \%$ from the baseline to the End of Project Evaluation. The Most probably contribution of the project to this reduction was through the implementation of community based Nutrition interventions through the Parent Support Group [7] (PSG) initiatives where the Project worked together with the community to encourage appropriate infant and young Child Nutrition including meal frequency, adequate meal preparation and utilisation of the Timed and Targeted Counselling (ttC) [8] model to follow up on parents to be able to feed their Children appropriately before they turn 2 years. This was in line with the current Scaling up of Nutrition (SUN) [9] initiative that has shown evidence that recommends projects to focus on appropriate Nutrition for children before two years to be able to contribute to the reduction in stunting levels

\subsubsection{Percentage Reduction in Anaemia in Pregnant Women}

According to the Ugandan Demographic and Health Survey (UDHS) 2011 [10], overall, 23 percent of women aged 15-49 are anaemic and because of the above prevalence, the $\mathrm{MoH}$ [11] recommend that all pregnant women need Iron-folic supplementation through the pregnancy period. The project therefore decided to support the implementation of the integrated community outreaches (ICO) that increased access to services by pushing them to the community and included Goal oriented ANC that promotes offering Iron Folic supplements to pregnant mothers as early as possible during their antenatal care (ANC) visits. In this regard, Women reported to have consumed Iron folic supplements during their pregnancy increased from $78.8 \%$ at the baseline to $92.9 \%$ at MTR and finally $91.4 \%$ at the ETR presenting an increment of $12.6 \%$ pregnant women during the project life span.

To be able to fight against anaemia during pregnancy, the $\mathrm{MoH}$ recommends that a multi-prong approach be implemented. One of these approaches is to prevent malaria during pregnancy as malaria also contributes to the prevalence of anaemia in pregnant women. Pregnant women can reduce the risk of adverse effects of malaria through the intermittent preventive treatment (IPT) consumption of fansidar and by sleeping under insecticide-treated mosquito nets. Again, through the ICO and the promotion of Goal Oriented ANC, the $2^{\text {nd }}$ and $3^{\text {rd }}$ trimester ANC visits offered an opportunity for pregnant women to consume IPT $1 \& 2$ respectively and led to a sustained high IPT consumption from $83.0 \%$ at the MTR to $85.5 \%$ at ETR. The high proportion attained at end of project evaluation was observed to be in line with the national HSSIP target of pregnant women who have completed IPT2 uptake increased from $42 \%$ to $80 \%$ by 2015 .

Further still during the baseline, $79.9 \%$ of the women from the project area reported use of long lasting insecticide treated nets (ITNs) for prevention of malaria. At the MTR, ownership of ITNs was reported by $67 \%$ of women, which increased to $86.7 \%$ at the ETR. This presented an increment of $7 \%$ from the baseline. The percentage increment is attributed to the Behaviour Change Communication (BCC) messages that were being passed on by the VHT during the implementation of the $\mathrm{ttC}$ model through the 1000 days period and was further noted to be in line with the national HSSIP target of the households with at least one ITN increased from $46.7 \%$ to $85 \%$ in 2015 .

\subsubsection{Percentage Increase in Number of Women Delivered by a Skilled Birth Attendant}

Some of the key interventions for preventing neonatal deaths revolve around the quality and package of care provided at the time of birth. It includes delivery of the baby by a skilled birth attendant, emergency obstetric care, and immediate care for the newborn baby, including breastfeeding support and resuscitation of the newborn. The recommendations from the World Health Organisation (WHO) [12] is for healthy mothers and newborns to receive care from the health facility for at least 24 hours after an uncomplicated vaginal birth at the facility. The project over the years implemented the $\mathrm{ttC}$ and part of the counselling support that the VHTs were offering to the Mothers was to plan for their birth and have it at the health facility. Further still, the project invested in strengthening the Health centres around care practices for the delivery through the Helping baby's breath (HBB) [13] and the baby Friendly health facility initiatives (BFHI) [14]. The proportion of women from the project area who reportedly delivered at the health facilities and by implication, under skilled service providers during the baseline was $91.3 \%$, increased slightly to $94.0 \%$ during the MTR and further at the ETR to $97.2 \%$. The results indicate a $5.9 \%$ increase in the number of women delivered by a skilled Birth attendant.

The finding was observed to be a big improvement from the situation at the project inception, when the UDHS 2011 reported overall $57 \%$ of births in the country had taken place at health facilities, and within the North region that includes 
Kitgum district it was at $52 \%$. It was also observed to be in line with the national HSSIP target of women who deliver in health facilities increased from $34 \%$ to $90 \%$ by 2015 . An important finding from the FGDs was the reported involvement of men in this area that was traditionally "offlimits" as reflected in the following statement:

"...Men now accompany their wives to the health centre and also give the money to prepare for delivery. They also attend meetings together..." [VHT Member from Mucwini Sub-county]

\subsection{Progress Towards Outcome One: Improved and Equitable Access to MNCH Services}

For the project to be able to contribute to the health system and be able to facilitate the processes of extending it to the community level to expand inclusiveness in all the processes, EAMNCH viewed the health system as a continuum of care providing quality $\mathrm{MNCH}$ services from the household to health facility and finally inputting into the Kitgum District health department annual work plans. For longer term sustainability, the project built local and district capacity of community and facility based elements of the health systems. It promoted dialogue between service providers and users to define entitlements, responsibilities and shared expectations. Communities were sensitized in order to increase their desire for quality MNCH services.

\subsubsection{Percentage Increase in Women Receiving 4 Quality Ante-Natal Visits}

Access to quality antenatal care is important in order to screen for the pregnancy related complications and provide appropriate interventions that result in positive birth outcomes for both the mother and baby. The $\mathrm{MoH}$ recommend at least four goal-oriented antenatal care visits for uncomplicated pregnancy, which start right from the first trimester. The proportion of women who made at least four antenatal care visits within the project area at baseline $80.8 \%$ of the pregnant women made the recommended minimum of four antenatal care visits but this declined to $75.8 \%$ during the MTR. However, the proportion increased to $82.4 \%$ during the end of project evaluation. This presents a $1.6 \%$ increase in the indicator. The percentage increase in this case seems to be minimal and one would ask why the project invested in ANC when its percentage was already above the national target of $60 \%$, however it's important to note that these communities were based in camps before and each camp had a health center so it was easy for women to visit these centers as many times as possible. The project therefore set out to sustain this rate even when the community had gone back to the villages. The Projects community $\mathrm{MNCH}$ behavioral change strategy of implementing the ttC model where the VHTs visited the households at least around the time they were scheduled for ANC visits, was used to encourage the pregnant mothers to actually make their visits in a timely manner, further still the monthly ICOs brought the goal oriented services closer to the communities and therefore helped the project to sustain a percentage above the national target and current average. The findings during ETR was noted to be in line with targets set under the HSSIP, the proportion of pregnant women attending ANC 4 times increased from $47 \%$ to $60 \%$ by 2015 .

\subsubsection{Percentage Increase in Male Involvement in the Goal Oriented ANC Services}

The national policy and guidelines promote active involvement of men in $\mathrm{MNCH}$ due to their dominant decision-making roles, the awareness that gender affects sexual behaviours and reproductive decision-making as well as overall health and well-being of the household. At the MTR, male partners or spouses were reported to have accompanied $72.3 \%$ of the women for $\mathrm{MNCH}$ related services at the service provision point and during the ETR, $90.4 \%$ of the women. This presents $18.1 \%$ increment. The findings were noted to be in line with what emerged from KIIs and FGDs which revealed that there had been observed progressively greater involvement of men in $\mathrm{MNCH}$ services, as reflected in the following statement by a participant:

"There is change...men are motivated to move with their women to the antenatal care clinics..." [PSG Member, Lamogo-Lagoro Sub-county]

The Project implemented PSG aimed at increasing the dialogue between the men and women at household level. The PSGs met at least once a month and the VHT in that community facilitated discussions around $\mathrm{MNCH}$ and the role that men are supposed to play in escorting their partners to either the ICO site or the Health facility. The PSG had a strategy to make every man accountable for another for involvement to happen. One of which was to accompany their wives for ANC care during the ICOs.

\subsubsection{Percentage Increase in Women Using Family Planning}

Table 1. Reported Ever Use of Family Planning within the Project Area in 2011, 2013 and 2016.

\begin{tabular}{llll}
\hline Year & $\mathbf{2 0 1 1}$ & $\mathbf{2 0 1 3}$ & $\mathbf{2 0 1 6}$ \\
\hline Percentage & $60.4 \%$ & $54.5 \%$ & $48.0 \%$ \\
\hline
\end{tabular}

Effective family planning enables individuals and couples to correctly space and time the births of their children as well as attain the desired number of children. The proportion of women with children age 0 to 23 months old who reported having ever used any family planning method within the 6 months preceding the baseline, MTR and ETR is presented in Table 1 . It revealed that ever use of family planning declined from $60.4 \%$ to $54.5 \%$ and to $48 \%$ respectively. This presents a percentage decrease of $12.4 \%$ for the project site.

Correct knowledge about family planning is an important requirement for adoption of correct attitude towards consistent use of the method. The health workers who provide $\mathrm{MNCH}$ services constitute an important source of this. The proportion of women who had reportedly received talks to influence their knowledge on family planning, at the ETR was $87.7 \%$ and these messages are given to them at the IOC, through the PSGs and the ttC visits conducted by the 
VHTs. The results indicate that the project employed as many channels as possible to educate and encourage the population to access and utilise family planning messages and commodities.

However further analysis at the ETR revealed that the family planning methods reportedly used by the women are: injectable, the most popular method, $43.2 \%$ followed by implants at $31.8 \%$, male condom $12.1 \%$ which was worrying given the fact that it is being promoted for the dual protection of sexually transmitted infections and unintended pregnancies. The other relatively popular method was intrauterine devices (IUD) 7.6\%.

\subsubsection{Percentage Increase in Children 0-59 Months Who Received Age Appropriate Immunization}

Table 2. Children from Project who Received DPT3 and Measles Vaccination in 2011, 2013 and 2016.

\begin{tabular}{lllll}
\hline \multirow{2}{*}{ DPT3 } & & $\mathbf{2 0 1 1}$ & $\mathbf{2 0 1 3}$ & $\mathbf{2 0 1 6}$ \\
& Yes, with Card & $82.20 \%$ & $81.80 \%$ & $76.80 \%$ \\
\multirow{2}{*}{ Measles } & Yes, without Card & $16.80 \%$ & $13.20 \%$ & $21.40 \%$ \\
& Yes, with Card & $73.90 \%$ & $71.40 \%$ & $73.80 \%$ \\
& Yes, without Card & $17.20 \%$ & $12.50 \%$ & $20.20 \%$ \\
\hline
\end{tabular}

Immunisation of children against the preventable diseases is among the high impact interventions to prevent child morbidity and mortality. The child in Uganda is fully immunized after receiving a single dose of the BCG vaccine, 3 doses of the Pentavalent (DPT $+\mathrm{HepB}+\mathrm{Hib}$ ), and one dose of the Measles vaccine at about 9 months. The proportion of children aged 6-23 months who received third dose of Pentavalent and measles vaccines during the baseline, MTR and ETR has been summarised in Table 2:

It revealed that at the baseline, $98.9 \%$ of the children had reportedly received the third dose of pentavalent vaccine but this could only be objectively verified using the child health card for $82.2 \%$. At the MTR, $94.9 \%$ of the children had been vaccinated with objective verification possible for $81.8 \%$ and at the ETR, $98.2 \%$ of the children had been vaccinated with verified with the child health card for $76.8 \%$.

The proportion of children who had received measles vaccine was $91.1 \%$ at baseline but verified with the child health card for $73.9 \%$, declined to $83.9 \%$ at MTR with verified for $71.4 \%$ of children, and increased again to $94 \%$ at the ETR with verified with child health card for $73.8 \%$.

Taking measles vaccination as an indicator of choice the percentage increase in children who were vaccinated fully stands at a $4 \%$. Immunisation is a national wide intervention and the project invested heavily in facilitating the access to immunisation services through the ICOs, acceleration through support of the child days plus $\mathrm{MoH}$ initiatives together with making sure that the children get the immunisation they need timely through the $\mathrm{ttC}$ household visits. This was to make sure that as the population resettles back into the community, access to the immunisation on schedule is maintained and therefore maintaining the high coverage. The national immunisation coverage targets set under the HSSIP are: the DPT-3/Pentavalent coverage for under 1's increased from $74 \%$ to $85 \%$ by 2015 ; and the measles vaccination coverage by 12 months of age increased from $75 \%$ to $95 \%$ and the project exceeded these targets.

\subsection{Progress Towards Outcome Two: Adoption of Positive Nutrition and WASH Practices}

Prevention of $\mathrm{MNCH}$ problems is better than curing them. For that reason a strong emphasis was on empowering the communities to care for themselves and their children. This included creating awareness on healthy nutrition for pregnant and lactating women as well as children, and enabling communities to produce and access sufficient nutritious food. It also involved providing the communities with knowledge and skills to access safe water and practice good hygiene and sanitation. The project embarked on this to contribute to the $\mathrm{MoH}$ Uganda Nutrition Action plan [15] that advises appropriate Nutrition for the 1000 days window for child survival through the ttC, PSGs, Stepping stones, aimed at challenging traditional and socially constructed beliefs that are harmful.

\subsubsection{Increase in Women of Reproductive Age Practicing WHO Standards for IYCF}

This outcome indicator facilitated the project to focus on community and Health facility practices that aim at giving colostrum through the early initiation of Breastfeeding, Exclusive Breastfeeding, appropriate and timely complimentary feeding focusing on the correct time in the 1000 days window period. At the Health facility level the project focused on implementing the $\mathrm{MoH}$ transformation of Health facilities to become mother-baby friendly model called the Baby Friendly Health Facility Initiate (BFHI). At the community level the project strengthened the PSGs through the Essential Nutrition Actions (ENA) initiates, ttC and implemented promotion of WASH practices through the stepping stones methodology.

\section{i. Early Initiation of Breastfeeding}

The MoH Uganda adapted the WHO and UNICEF [16] recommendation of initiation of breastfeeding within one hour of the baby's birth, exclusive breastfeeding for the first six months of the baby's life, and continued breastfeeding up to 2 years of age and beyond with adequate and appropriate complementary food starting from 6 months.

The proportion of children aged 0-23 months who were reportedly initiated to breastfeeding within one hour of birth baseline was $73.8 \%$ which increased slightly to $75.8 \%$ at MTR. However, this was followed by a decline to $67.3 \%$ at ETR. Basing on these results the project instead reported a decreasing trend of $6.5 \%$ from the baseline.

However it's important to note that the project invested lots of community interventions into this outcome both at the Health facility and community Level. Further still particularly for this section the questions agreed at the ETR processes was asked differently from the one aske both at baseline and MTR which could have probably contributed to the decline in the percentage contribution. At the project inception UDHS 2011 
reported at national level $52.5 \%$ of the children started breastfeeding within one hour of birth and more specifically, within the North region $38.4 \%$ of children were initiated within the first hour. Basing on this revelation, the project still celebrates a high percentage than the national average.

\section{ii. Exclusive Breastfeeding}

The proportion of children aged 0-23 months who were reported to have been exclusively breastfed for the first six months of life at the baseline was $72.1 \%$ and increased to $82.7 \%$ at MTR. However, declined to $78.9 \%$ during the ETR. This presents a $6 \%$ increment from the baseline. This should have been a much better contribution basing on the fact that the project invested evidence based models at the household level to make this a reality. However as explained in the early initiation scenario, this indicator question was also changed at the ETR and this could have contributed to the low rates. Even with this decline from the MTR, the finding at end of project evaluation was observed to be quite in line with the national HSSIP target of increased exclusive breastfeeding at 6 months from $60 \%$ to $80 \%$ by 2015 .

\section{iii. Complementary Feeding}

The national Infant and Young Child Feeding (IYCF) [17] guidelines recommend introduction of solid and liquid foods (complementary foods) to infants around the age of 6 months because the breast milk alone is no longer sufficient to maintain the child's optimal growth. The timeliness in introduction of complementary foods among the infants at the MTR, complementary feeding was initiated at around six months of age for $76.4 \%$ but declined to $60.3 \%$ at ETR. The decline could be attributed to increase in proportion of infants who were initiated to complementary feeding before 5 months of age, from $7 \%$ to $35.9 \%$. The finding at ETR was observed not to be in line with the national HSSIP target of timely complementary feeding increased from $73 \%$ to $80 \%$ by 2015 .

\subsubsection{Increase in Households That Wash Hands with Soap After Visiting the Toilet}

Good household level water, sanitation and hygiene practices contribute towards improved health of mothers and children. The evaluation explored the following areas: proportion of households that reported collection of drinking water from unprotected sources; the proportion of households that reported no toilet facilities or the use of bush; and proportion of respondents that reported use of soap after visiting the toilet.

$87.7 \%$ of the households at baseline obtained drinking water from protected sources but this declined to $81.8 \%$ at MTR and increased to $91.7 \%$ at ETR. A similar pattern of a slight decline from the baseline $(76.6 \%)$ to the MTR (75.1\%), followed by an increase at ETR (78.8\%) was observed for use of toilet facilities.

Availability of hand washing facility was reported by $25.7 \%$ of households at the baseline, and at MTR $81 \%$, which increased to $83.8 \%$ at ETR. This presents a $58.1 \%$ increment from the baseline. This percentage increase is attributed to the Stepping stones initiative that concentrated on community Hygiene and sanitation in the project area.

The relevant targets set within the HSSIP were: the households with hand washing facilities with soap increased from $22 \%$ to $50 \%$ by 2015 ; and households with pit latrines increased from $67.5 \%$ to $72 \%$ by 2015 . Findings on improved household level water and sanitation hygiene was confirmed from the FGDs and KIIs as exemplified by the following statement:

"People now appreciate the use of toilets and tip-taps due to sensitization through project activities unlike before where people used to construct latrines but did not use them..." [Community Leader, Lagoro Sub-county]

\subsection{Outcome Three: Favorable Policy Environment for Improved $\mathrm{MNCH}$}

The project worked with communities to: increase information transparency and accessibility; strengthen their voice through education and empowerment; provide opportunity for dialogue; and encourage power holders to be accountable. The project worked to build community advocacy capacity using the CVA [18] approach. As issues emerge through this organic approach, driven by community priorities, community voices were amplified by feeding into the Child Health Now [19], WV's campaign platform for advocacy work on MNCH issues at national and international level. These voices provided a platform for World Vision Uganda's contribution to the Uganda Civil Society for Scaling up Nutrition efforts that led to the development of the Uganda Nutrition Action Plan, The Uganda Civil Society for maternal Newborn and Child Health efforts that contributed to the Reproductive, Maternal Newborn and Child Health Sharpened plan efforts.

\subsubsection{Percentage Increase in Community Groups That Demand Better MNCH Services}

The project led to the creation of 2 CVA groups in the implementation area, these were non-existent before the project inception. The CVA local groups facilitated the local community dialogues and escalated the issues to the subcounty dialogues. Some of the issues that could be handled by the sub-counties were assimilated into the sub county action plans and those that needed the intervention of the district were escalated to the district by the same teams.

\subsubsection{Build Community Advocacy Capacity}

The Two CVA groups that the project facilitated to be generated included 8 women and 30 men. These groups contributed to access to $\mathrm{MNCH}$ services by working through the PSGs whose capacity was built by the project to construct 18 outreach huts in areas where the project was facilitating ICO. These outreach huts were instituted after the community discussed the issue of privacy for the Women during the ANC and PNC sessions under the mango tree, and the fact that during the rainy seasons the outreaches could not take place in an open place. 


\section{Discussion of Results}

The EAMNCH project facilitation to the Kitgum District Health department was aimed at making sure that the key $\mathrm{MNCH}$ indicators in the two sub counties of Lagoro and Mucwini improve. The key entry point for this to happen was through a few evidence based and low cost models that have proven to offer sizeable contribution and improvement to the indicators. Four key approaches were instituted and they account for most of the successes as portrayed in the improvement. Their contribution is as stated below:

\subsection{Collaboration and Consultations with the District, Health Systems Strengthening Approaches}

The Projects contribution to the improvement to equitable and access to $\mathrm{MNCH}$ services, at the Health facility the efforts invested into a joint capacity building plans for the key contact points service providers. The projects capacity needs assessment in $\mathrm{MNCH}$ necessitated the need to build capacity in key MoH Ugandan packages including the HBB, the BFHI and Helping Mothers survive to improve skills of Health Workers in offering efficient MNCH services. The project also invested in technical support supervision, mentorship and period coaching and offering period feedback to the health centre Team. This EAMNCH project implementation strategy to improve $\mathrm{MNCH}$ in Kitgum through health system strengthening is a contribution to the WHO global strategy of strengthening health outcomes through system approaches [20]. The support was offered through a collaborative approach where the technical support was being physically offered by staff from the MoH jointly with the District Health Team. These are the supervisors of the health facility staff and therefore promoting interaction between the staff and the supervisors focusing on the actual skills required for the Health centre staff to offer equitable services to the mothers and children in the project area. With this the project evidently recorded an improvement in delivery at the Health facility, and essential newborn care and Past Natal care. The skills passed on have also contributed to the sustainability plan of making sure that all staffs have the required skills to deliver Health services even when the project exited.

\subsection{Community Health Systems Appraisal}

The project registered an overall improvement in the Attandance of 4 ANC visits, appropriate Hygiene and sanitation practices at the household level, improvements around the Essential household $\mathrm{MNCH}$ practices, and male involvement into $\mathrm{MNCH}$ services. These are some of the key deliverables that the Community Health Workers were tasked with facilitating. The Ugandan VHTs operating at the household level, needed to be empowered to be able to offer services that contributed to the improvement of the indicators. World Vision Uganda being a Community based Organisation fast tracked the strengthening of the VHT system at the project level to be able to equip and empower the VHTs to deliver. The strengthening of the VHT system in the project was jointly spearheaded by the Project and the District Health department, an assessment looking into the functionality of the VHTs in the project area was done at the onset of the project and the key gaps contributed to the targets set for input into this system. There was periodic joint monitoring of the progress of this functionality that involved, the District Health Team, the other stakeholders that invested in VHTs and the VHTs themselves. This also contributed to the investment in the right gaps and capacity needs, and prevented duplication of efforts since other stakeholders were also involved and therefore provided updates on where they are and what they are investing funding currently. The process also motivated the VHTs to do their quality Household walks, update their registers, and offer household counselling and referral to the health facility for early care.

\subsection{Households Empowerment and Demand for Health Services}

The project set out to empower households to be able to understand the $\mathrm{MNCH}$ services that they require at a particular time, and these households were further empowered through the CVA to actually pressure service providers to offer equitable services. The strategy was aimed at making household members get to the understanding that Health is made at home and knowledge about how to access health care is key for the households to eliminate any delays that may result into morbidity and mortality. This is agreement with a study [21], where it was found out that Programmatic policies in overcoming barriers to access are a useful factor in influencing community demand for health services. The approach of having both women and men have a discussion around a MNCH care related topic and jointly come up with a solution facilitated the elimination of barriers that are gender related. It is however important to note that the project registered a reduction in the access and utilisation of family planning services, even when the project had a focus on empowering families to agree to Health Timing and spacing pregnancies. However, the project registered improvement in male involvement into $\mathrm{MNCH}$ services, and other household based Young Child practices that are related to household empowerment.

\subsection{Low Cost but Effective Models Implementation}

The Project invested in a number of community based models, these were aimed to improve as much knowledge as possible for households, facilitate timely response of parents to their children in terms to growth monitoring and promotion, timely visitation for goal oriented ANC services, delivery at the Health facility and timely referral in case of danger signs. The implementation of the $\mathrm{ttC}$ model was in line with the evidence based support that the project needed to offer for the 1000 days principles, the PSGs was aimed at empowerment of couples to take joint responsibility for $\mathrm{MNCH}$ related issues in the household and focus on Nutrition care and support. The Infant and young Child 
feeding indicators related to the Women of reproductive age practicing exclusive breastfeeding, early initation of Breastfeed and timely complementary feeding are evidence to show that actually investment into PSGs worked. For the services like complete immunization, Goal oriented ANC, and uptake of the Iron folic supplementation are strongly related to the implementation of the ICOs in communities. The project together with the Health facility teams scheduled the ICOs to bring these services closer to the community and therefore promote $\mathrm{MNCH}$ services uptake. Lastly the project implemented the stepping stones for behavioral change around the WASH initiatives and as seen in the outcomes, the project registered the highest success on the WASH indicators.

\section{Conclusion and Recommendations}

\subsection{Conclusion}

Implementation of the planned interventions under the EAMNCH Project contributed towards the set goal of improving $\mathrm{MNCH}$ in Lagoro and Mucwini sub-counties. Evidence from this supported the contribution of project interventions to the reduction in infant and under-five child morbidity as well as mortality within the project area. It also supported the contribution from the project interventions towards reduction in maternal morbidity as well as mortality within the project areas. However, there was less successful results obtained in the area of infant and young child feeding, with particular reference to complementary feeding practices. The observed decline in some indicators could be attributed to issues related to the apparent decline in quality of services provided at health facilities as evidenced by high staff turnover and sociocultural factors that hindered the uptake of services such as family planning.

\subsection{Recommendations for Future Projects}

Implementation of the EAMNCH in Kitgum gave rise to a number of key programmatic recommendations including the following:

(1) The relatively new model of work that involved diversion from direct service delivery to strengthening of existing health systems and working in partnership with local government and other NGOs could yield positive and sustainable results. For instance, where the Health Management Information System (HMIS) was weak, the project focussed on its improvement. This led to better quality data and planning, particularly since the project M\&E system was reliant upon HMIS for data related to uptake of services from health facilities. This if replicated or scaled up could be a big support to the Ugandan Health system.

(2) The adoption of the integrated approach to service delivery resulted in a comparatively more complex project, which called for optimal administrative and logistic support as well as staffing in order to ensure good quality results from the implementation. It's therefore recommended that for further programming there is need to include a human resources for health component, this if combined with the System's strengthening approach would go a long way to contribute to sustainability.

(3) On-job mentorship and coaching for service providers was a practical approach that could be effectively utilised to address identified gaps in the capacity of health workers to deliver quality MNCH services. This would go a long way if replicated or scaled up but in addition to strengthening the quality improvement and assurance for $\mathrm{MNCH}$ through the contact points using the District Health Team MNCH members.

(4) The approach of active involvement of men in the PSGs highlighted them as role models, greatly influenced the gender dynamics and resulted in more support to their wives as well as rapid uptake of $\mathrm{MNCH}$ services. This is a best practice and should be scaled up in promotion of having men on board for Maternal Health

Despite the positive lessons from the project implementation, the key informants highlighted some of the lost opportunities, as follows:

(5) Mobile Health (mHealth) was recognised at project inception to provide the opportunity for innovation due to the strong potential to influence $\mathrm{MNCH}$ programming. However, the bottlenecks confronted proved to be an obstacle for its inclusion within the project framework and timeline.

(6) Implementation Research was identified at inception as a critical area that would contribute towards project learning. However, operationalization was fraught with challenges that made it eventually impossible to attain the expected results within the project lifetime.

(7) Retention and Motivation of the community volunteers and support group members proved to be a challenge particularly since replacement involved re-investment in training and related capacity building activities.

\section{Acknowledgement}

The East African $\mathrm{MNCH}$ project was implemented with Donations from the Australian Government, Department of Foreign Affairs and Trade as part of the Australia Africa Community Engagement Scheme (AACES) through the World Vision Australia office. Funding was utilised by World Vision Uganda in partnership with the Kitgum District Local government from 2011 to 2016

\section{References}

[1] Republic of Uganda, National population and housing census 2014 report.

[2] Kitgum District Development Plan (DDP), 2008/09. Uganda.

[3] "As if we weren't human" Discrimination and Violence against Women with Disabilities in Northern Uganda, Human Rights Watch, August 2010. 
[4] Uganda Aids Atlas, Ministry of Health, 2005.

[5] Australian Government, Department of Foreign Affairs and Trade: Australia Africa Community Engagement Scheme (AACES) Program, 2011, http://dfat.gov.au/aboutus/publications/Pages/aaces-program-review-2011-2016.aspx.

[6] The Uganda HEALTH SECTOR STRATEGIC \& INVESTMENT PLAN: Promoting People's Health to Enhance Socio-economic Development, 2010/11-2014/15, Ministry of Health.

[7] Gloria K. Seruwagi et el: Investigating Associations between Parent Support Groups and Integrated Outreach Services for Maternal and Infant Survival: Experiences from a Project in Northern Uganda, 2015. http://sciencedomain.org/issue/1196.

[8] World Vision International: TIMED AND TARGETED COUNSELLING FOR HEALTH \& NUTRITION, 2014, http://www.wvi.org/sites/default/files/Participants\%20Manual $\% 20$ (TTC)\%20METHODOLOGY.final_.pdf.

[9] Scaling Up Nutrition (SUN) Movement Strategy [2012-2015] https://scalingupnutrition.org/wpcontent/uploads/2012/10/SUN-MOVEMENT-STRATEGYENG.pdf.

[10] Uganda Demographic and Health Survey, 2011.

[11] The Republic of Uganda, REPRODUCTIVE MATERNAL, NEWBORN AND CHILD HEALTH SHARPENED PLAN FOR UGANDA, November 2013. Ministry of Health.

[12] WHO, 2014. Recommendations on Postnatal Care of the Mother and Newborn.
[13] Ministry of Health, Uganda, Helping babies Breath Plus, Facilitators Flip Chart, 2015.

[14] Ministry of Health, Uganda, BREASTFEEDING PROMOTION, PROTECTION AND SUPPORT IN A BABY-FRIENDLY HEALTH FACILITY, 2010.

[15] Ministry of Health, Uganda, UGANDA NUTRITION ACTION PLAN $2010-2015$.

[16] WHO: Infant and young child nutrition, Global strategy on infant and young child feeding, 2002, http://apps.who.int/gb/archive/pdf_files/WHA55/ea5515.pdf? ua $=1$.

[17] Republic of Uganda, MoH, Policy Guidelines on Infant and Young Child Feeding, 2009.

[18] World Vision international, Citizen Voice and Action, 2012. http://www.wvi.org/sites/default/files/Citizen_Voice_and_Acti on_PM.pdf.

[19] World Vision International, CHILD HEALTH NOW TOGETHER WE CAN END PREVENTABLE DEATHS, 2009. http://www.wvi.org/sites/default/files/child-health-nowreport_1.pdf.

[20] WHO (2007), Everybody's Business: Strengthening health systems to improve health outcomes, WHO framework for action; Geneva-Switzerland, 1-56.

[21] Tim E and Stephane C, 2004, overcoming barriers to health services: influencing the demand side, International Programme, Centre for health Economics, New York; Health Policy and Planning Journal 19 (2), 69-79. 